\title{
Concurrent Program Verification With Invariant-guided Underapproximation
}

\author{
Sumanth Prabhu $\mathrm{S}^{1}$, Peter Schrammel ${ }^{2}$, Mandayam Srivas ${ }^{1}$, \\ Michael Tautschnig ${ }^{3}$, and Anand Yeolekar ${ }^{4}$ \\ 1 Chennai Mathematical Institute, Chennai, India, \\ ${ }^{2}$ University of Sussex, Brighton, UK, \\ 3 Queen Mary University of London, UK, \\ 4 Tata Research Development and Design Centre, Pune, India
}

\begin{abstract}
Automatic verification of concurrent programs written in low-level languages like ANSI-C is an important task as multi-core architectures are gaining widespread adoption. Formal verification, although very valuable for this domain, rapidly runs into the state-explosion problem due to multiple thread interleavings. Recently, Bounded Model Checking (BMC) has been used for this purpose, which does not scale in practice. In this work, we develop a method to further constrain the search space for BMC techniques using underapproximations of data flow of shared memory and lazy demand-driven refinement of the approximation. A novel contribution of our method is that our underapproximation is guided by likely data-flow invariants mined from dynamic analysis and our refinement is based on proof-based learning. We have implemented our method in a prototype tool. Initial experiments on benchmark examples show potential performance benefit.
\end{abstract}

\section{Introduction}

Automatic verification of concurrent programs written in low-level languages like ANSI-C is an important task as multi-core architectures are gaining widespread adoption. Difficulty in development of programs due to concurrency and different memory models of processors underlines the need for tool support. Bounded Model Checking (BMC) has been proposed as a solution for this purpose which tries to ferret shallow bugs limiting unwinding depth [1], number of contextswitches [2], or number of writes [3, 4] as a bounding parameter to restrict search space and manage complexity. In these techniques, the control flow of the concurrent program is sequentialized by choosing an arbitrary thread order, and then modeling the effect of all interleavings by symbolically encoding the possible read-write partial orders as non-deterministic data-flow constraints on all behaviors up to the chosen BMC bounding parameter.

Contributions. In this work, we develop a method to further restrict proof search space by using semantic underapproximations of possible data flow (i.e., write-to-read relations in happens-before orders) of shared memory accesses and lazy, on-demand refinement of the approximation. The novel contributions of our work are the following: 
invariants on data flow following [6]. For instance, in most executions of the program in Fig. 1 , reads at $t 1_{4}$ and $t 1_{7}$ will refer only to the local write $\left(t 1_{5}\right)$ due to the spin-lock like condition in $t 2_{3}$.

If the set of possible executions of a given program is represented as $L_{C} \cap$ $L_{D}$, where $L_{C}$ is the set of executions from the control-flow graph and $L_{D}$ is the allowed data flow in underlying memory model, then our tool starts with $L_{C} \cap L_{D^{\prime}}$, where $L_{D^{\prime}} \subseteq L_{D}$. It either proves that $L_{D} \backslash L_{D^{\prime}}$ is irrelevant to the property or unfeasible, or refines $L_{D^{\prime}}$ towards $L_{D}$. This has the advantage that if the program is unsafe in the restricted model (as shown on Fig. 1) then we can find a counterexample earlier; otherwise we explore data flows which are only relevant to the property. To construct an initial $L_{D^{\prime}}$ such that $L_{D^{\prime}} \subseteq L_{D}$ we use likely invariants on data flow following [6].

We use unsatisfiable cores produced by a SAT solver for refinement of our data-flow invariants on a demand-driven basis. Our refinement algorithm works as shown in Fig. 2. We start with a Boolean formula, which is constructed by converting the conjunction of given program $(P)$, negation of a property $(\phi)$ and a set of constraints (Inv $:=I_{1} \wedge \ldots \wedge I_{n}$ ) to Boolean form (CNF) using an appropriate method (like bit-blasting). This yields an underapproximation of the original formula $(P \wedge \neg \phi)$, which is passed to a SAT solver. If the formula is satisfiable then we deduce that the input program is unsafe. If, however, the formula is unsatisfiable then we check whether any of $I_{1} \ldots I_{n}$ from Inv are part of the unsatisfiability proof (unsatisfiable core) $C$. If none of the clauses originating from $I_{1} \ldots I_{n}$ are present in $C$ then we decide that the input program is safe. Otherwise we consider Inv $:=\left\{I_{1}, \ldots, I_{n}\right\} \backslash C$ for the next iteration.

Lemma 1. Soundness: If our algorithm terminates with the outcome "Safe", then the property $\phi$ is guaranteed to hold; if it terminates with "Unsafe" then $\phi$ is violated.

Proof: In symbolic BMC, the program and the assert predicate is converted to a Boolean formula of the form $P \wedge \phi$, where $\phi$ is the negation of the asserted predicate. To this formula we conjoin additional constraints $I_{1} \wedge \ldots \wedge I_{n}$ to get a Boolean formula $\underline{P}:=P \wedge \phi \wedge I_{1} \wedge \ldots \wedge I_{n}$. Here $P, \phi$ and $I_{1} \ldots I_{n}$ are in CNF. We declare a given program as Unsafe when $\underline{P}$ is satisfiable. It is easy to see that if $\underline{P}$ is satisfiable then so is $P \wedge \phi$. This implies that $P \wedge \phi$ is also satisfiable and hence program is unsafe as $\phi$ is the negation of the asserted predicate.

We mark a given program as Safe when $C \cap\left\{I_{1} \ldots I_{n}\right\}=\emptyset$, where $C$ is an unsatisfiable core. By definition, $C$ is unsatisfiable and $C \subseteq \underline{P}$. Therefore we conclude that $C \subseteq(P \wedge \phi)$ as $C \subseteq \underline{P}$ and $C \cap\left\{I_{1} \ldots I_{n}\right\}=\emptyset$. Since $C$ is unsatisfiable $P \wedge \phi$ is also unsatisfiable as $P$ and $\phi$ are in CNF.

This proves the soundness of our algorithm.

Lemma 2. Completeness: Our algorithm always terminates for a finite-state concurrent program.

Proof: We start with $\underline{P}:=P \wedge \phi \wedge I_{1} \wedge \ldots \wedge I_{n}$. At each iteration we either decide safety of a program or consider $\left\{I_{1} \ldots I_{n}\right\} \backslash\left(C \cap\left\{I_{1} \ldots I_{n}\right\}\right)$, where $C$ is 


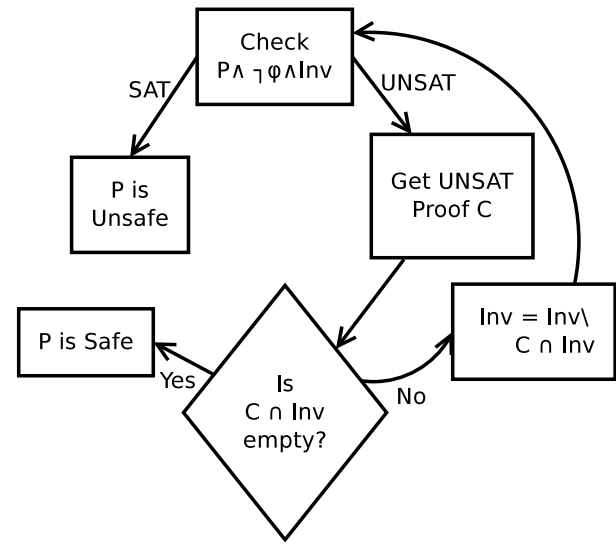

Fig. 2. Refinement flowchart

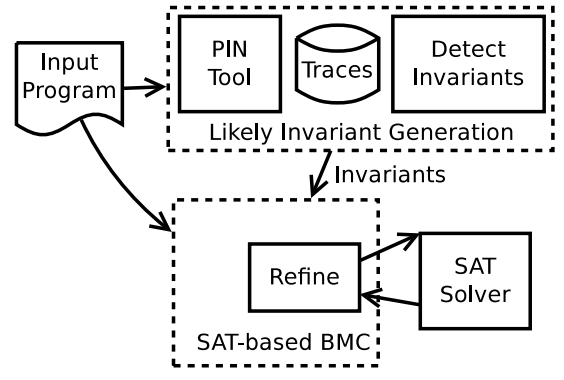

Fig. 3. Design of the tool

an unsatisfiable core. If we proceed without deciding about safety of the given program we will have $\underline{P}:=P \wedge \phi$ after a maximum of $n$ iterations. This formula is the original formula which can be decided. Hence, we always terminate in at most $n+1$ iterations.

\section{Implementation}

Our tool operates in two stages, shown in Fig. 3. First, likely invariants are generated by dynamic analysis, which are used to construct an underapproximation of the input program. In the second stage the tool performs SAT-based bounded model checking and refinement on the underapproximated input program. In subsequent sections we provide details of each stage.

\subsection{Likely Invariant Generation and Constraints}

The compiled input program is passed to binary instrumentation built using PIN [5]. We instrument shared memory instructions to collect execution traces, which are analyzed to detect three classes of likely invariants following [6]. The generated invariants are sequences of tuples where each tuple consists of a location of the instruction in the source code, the name of the variable on which the instruction operates, and the type of instruction (read or write).

These invariants are passed to CBMC, together with the input program and unwinding depth, via newly added options. Option --refine-cpu indicates to CBMC to invoke our changed code path. Options --invariant-strategy $l$ and --invariant-file file-name specify that likely invariants are to be read from filename for underapproximation. These likely invariants are considered while constructing the $r f$ relation: for reads appearing as likely invariants only writes that are present in the corresponding definition set are considered. In order to fall back to the original rf relation during refinement we add a switch variable while constructing the $r f$ relation, which, when disabled, yields the original $r f$ relation. For example, we construct the following formula: switch ${ }_{v_{1}} \Rightarrow$ $\left(r_{v_{1}}=w_{v_{1}}^{i_{1}} \vee w_{v_{1}}^{i_{2}} \vee \ldots w_{v_{1}}^{i_{m}}\right) \wedge \neg$ switch $_{v_{1}} \Rightarrow\left(r_{v_{1}}=w_{v_{1}}^{1} \vee w_{v_{1}}^{2} \vee \ldots w_{v_{1}}^{n}\right)$, where 
$r_{v_{1}}=w_{v_{1}}^{1} \vee w_{v_{1}}^{2} \vee \ldots w_{v_{1}}^{n}$ is the original $r f$ relation, $w_{v_{1}}^{i_{1}} \ldots w_{v_{1}}^{i_{m}}$ are writes corresponding to $r_{v_{1}}$ in the definition set and $w_{v_{1}}^{1} \ldots w_{v_{1}}^{n}$ is the actual set of writes in the program. These constraints along with the unwound program and property are converted to a Boolean formula [1].

\subsection{Refinement}

We have implemented the refinement algorithm of Fig. 3 in CBMC. Initially, all switch variables switch $_{v_{i}}$ are true. These constrain the $r f$ relation as seen in Section 3.1, and will act as constraints $I_{1} \ldots I_{n}$. We pass the Boolean formula constructed above to a SAT solver, which has the capability of generating an unsatisfiability proof. If the program is decided to be unsafe, a counterexample is returned. Otherwise we perform refinement as explained earlier.

\section{Experiments}

Our experiments address the following questions:

1. How effective are likely data-flow invariants in reducing the proof search space for verification? We measured the number of considered writes with our approach relative to the total possible writes of an unconstrained proof.

2. Does such a reduction in search space translate to a reduction in verification run time? We measure the SAT solver's time spent on a proof.

A reasonable question to ask related to the second item above is why can one expect SAT solver time to reduce by constraining proof search space. Note that we constrain search space by adding additional constraints (invariants) to the formula sent to the solver. Typically the distribution of solving times over degrees of constraining has a peak in the middle of the spectrum. That is, problems that are either under-constrained or over-constrained are easy because solvers encounter few conflicts. The latter because solver gets a solution mostly by propagation, the former because you get a solution mostly by making decisions only. The idea of adding additional constraints is to get us out of the middle of the peak towards the over-constrained side, which should make it easier for the solver although the formula is larger in size.

We ran our our tool on a set of targeted benchmark programs as well as benchmark programs from SV-COMP (pthread and pthread-atomic directories). The targeted benchmarks were constructed based on concurrent algorithms that had interesting data-flow invariants, e.g., programs that exhibited a large number of writes in possible atomic sections and different properties. Our experiments were run on a system with an i3 CPU $(1.70 \mathrm{GHz})$ and $4 \mathrm{~GB}$ RAM, running GNU/Linux OS. Our tool, the benchmark programs, and instructions to repeat our experiments are available in public [7]. For mining invariants, every program was executed to completion on random inputs and random interleaving for up to 50 execution traces. Since invariants were mined on limited runs there is no a-priori guarantee that they were true invariants. Table 1 shows the results corresponding to the targeted benchmark programs. We have experimented with both safe and unsafe programs and different unwindings as shown in columns labeled Type and U. The column Writes Saved indicates the total number of writes that 
were not considered when compared to the original encoding of CBMC. This is measured by taking the difference between the total number of writes that is considered in CBMC and the total number of writes considered with constraints for all reads. This will be 0 if we fall back to the original model after refinement (for example, 7.c). The Refinement columns indicate the number of constraints added in the beginning, the number of constraints remaining when a decision was taken, and the total number of iterations completed. The overall time taken by the SAT solver for CBMC and our tool with likely invariants encoded as constraints, as explained in Section 3.1, are shown in columns $C B M C$ and $L I$, respectively.

Our main observations are:

1. In all our targeted cases the mined invariants have been effective in reducing the proof search required to be considered as indicated by the numbers in the Writes Saved column. This shows that use of good invariants can have a potential impact in reducing proof complexity.

2. There has been a gain in speed in roughly half the number of cases (shown as bold face entries in File in Tab. 1).

3. However, the effect of the underapproximations on the reduction of the SAT solver time has been less significant. In some cases, we have observed that the SAT solver is slowed down even when there has been a significant reduction in the number of writes.

How can one explain observations 2 and 3, especially 3? SAT solver time is function of size of the formula as well as the number of variables. The formula representing the underapproximation is usually much larger (in terms of number of clauses) than the original model, which is one possible explanation for observation 3. To get evidence in support of this explanation, we constructed the underapproximated model more directly by eliminating the unnecessary writes at the partial-encoding itself (instead of adding them as clauses) resulting in smaller formulas. The NoR column shows the SAT numbers when run on this directly encoded model. As the numbers indicate this method of encoding reduces SAT time in most cases. There were a few exceptions shown by numbers in italics font. ( $T O$ indicates more than 200s.) One disadvantage of using this encoding is that it is not amenable for easy refinement.

Our results [8] on the SV-COMP benchmarks were mixed and not as good as for the targeted set. Since most SV-COMP benchmarks are stripped down to their minimal functionality, (1) the total number of memory accesses themselves were very small in most examples and (2) our dynamic analysis step produced very few invariants that could be used to cut down the partial read-write orders.

\section{Conclusions and Future Work}

We have developed a sound and complete tool to formally verify concurrent ANSI-C programs by automatically constructing underapproximations using likely data-flow invariants and incrementally refining them to get efficient proofs.

Our experimental results show that the tool can lead to reductions in proof search space and verification time on programs the synchronized behaviors of 


\begin{tabular}{|c|c|c|c|c|c|c|c|}
\hline File & Type & U & CBMC & LI & Refinement & Writes Saved & NoR \\
\hline 1.c & Unsafe & 10 & $14.06 \mathrm{~s}$ & $13.541 \mathrm{~s}$ & 87 to 87 in 1 & $1235 / 2390$ & $21.719 \mathrm{~s}$ \\
2.c & Unsafe & 10 & $2.835 \mathrm{~s}$ & $2.034 \mathrm{~s}$ & 28 to 28 in 1 & $450 / 912$ & $1.734 \mathrm{~s}$ \\
3.c & Unsafe & 20 & $21.127 \mathrm{~s}$ & $10.359 \mathrm{~s}$ & 58 to 58 in 1 & $1900 / 3727$ & $16.87 \mathrm{~s}$ \\
4.c & Safe & 16 & $39.633 \mathrm{~s}$ & $23.987 \mathrm{~s}$ & 107 to 88 in 5 & $1266 / 4489$ & $6.818 \mathrm{~s}$ \\
5.c & Unsafe & 16 & $28.273 \mathrm{~s}$ & $34.923 \mathrm{~s}$ & 93 to 93 in 1 & $2415 / 3710$ & $5.813 \mathrm{~s}$ \\
6.c & Unsafe & 21 & $15.984 \mathrm{~s}$ & $11.416 \mathrm{~s}$ & 42 to 42 in 1 & $1720 / 3144$ & $12.832 \mathrm{~s}$ \\
7.c & Safe & 6 & $48.716 \mathrm{~s}$ & $44.519 \mathrm{~s}$ & 22 to 0 in 4 & $0 / 599$ & $0.598 \mathrm{~s}$ \\
8.c & Unsafe & 11 & $4.567 \mathrm{~s}$ & $5.909 \mathrm{~s}$ & 32 to 32 in 1 & $685 / 1194$ & $5.41 \mathrm{~s}$ \\
9.c & Unsafe & 10 & $31.835 \mathrm{~s}$ & $17.196 \mathrm{~s}$ & 76 to 76 in 1 & $2115 / 3060$ & $8.553 \mathrm{~s}$ \\
$\mathbf{1 0 . c}$ & Unsafe & 10 & $101.484 \mathrm{~s}$ & $29.699 \mathrm{~s}$ & 76 to 76 in 1 & $1935 / 3060$ & $T O$ \\
$\mathbf{1 1 . c}$ & Unsafe & 9 & $38.624 \mathrm{~s}$ & $20.81 \mathrm{~s}$ & 83 to 83 in 1 & $1744 / 2868$ & $16.439 \mathrm{~s}$ \\
12.c & Unsafe & 9 & $62.895 \mathrm{~s}$ & $155.681 \mathrm{~s}$ & 68 to 68 in 1 & $1935 / 3060$ & $3.03 \mathrm{~s}$ \\
13.c & Safe & 10 & $7.392 \mathrm{~s}$ & $10.993 \mathrm{~s}$ & 22 to 8 in 3 & $144 / 736$ & $8.4 \mathrm{~s}$ \\
\hline
\end{tabular}

Table 1. Result of experiment on targeted benchmarks

which significantly constrain the possible read-write-orders that can be captured in the form of data-flow invariants. Producer-consumer-like programs, where consumers can only read from producers on a priority-based schedule, is one example that exhibits this characteristic. Our future work is aimed at eliminating some of the bottlenecks: (1) Alternate methods to encode the invariants without increasing size of the formulas, (2) Integrate an overapproximation step during refinement. (3) Interface with proficient open-source invariant mining tools. In related work, the use of underapproximations using number of of interleavings as a refinement metric was proposed in [9]. Distinction of our work is in the use likely invariants for this purpose.

\section{References}

1. Alglave J, Kroening D, Tautschnig M. Partial orders for efficient bounded model checking of concurrent software. CAV. 2013.

2. Qadeer S, Wu D. KISS: keep it simple and sequential. ACM SIGPLAN. 2004.

3. Tomasco E, Inverso O, Fischer B, La Torre S, Parlato G. Verifying concurrent programs by memory unwinding. TACAS. 2015.

4. Yeolekar A, Madhukar K, Bhutada D, Venkatesh R. Sequentialization Using Timestamps. TAMC. 2017.

5. Luk CK, Cohn R, Muth R, Patil H, Klauser A, Lowney G, Wallace S, Reddi VJ, Hazelwood K. Pin: building customized program analysis tools with dynamic instrumentation. ACM SIGPLAN. 2005.

6. Shi Y, Park S, Yin Z, Lu S, Zhou Y, Chen W, Zheng W. Do I use the wrong definition?: DeFuse: definition-use invariants for detecting concurrency and sequential bugs. ACM SIGPLAN. 2010.

7. https://github.com/sumanthsprabhu/atva_tool

8. http://www.cmi.ac.in/\%7Esumanth/dokuwiki/doku.php?id=invariants: underapproximation: experiments\#sv-comp

9. Grumberg O, Lerda F, Strichman O, Theobald M. Proof-guided underapproximation-widening for multi-process systems. ACM SIGPLAN. 2005. 